\title{
Clinical manifestations, complications, and outcomes of patients with COVID-19 in Sudan: a multicenter observational study
}

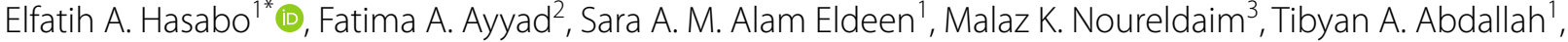 \\ Yousra T. Ahmed ${ }^{4}$, Safaa Badi ${ }^{5}$, Waleed K. Khalid ${ }^{6}$, Hind A. Mirghani ${ }^{2}$, Yossra A. Mohammed ${ }^{7}$, \\ Lubaba A. Alkhider', Saada A. Hamad', Amna M. Tagelseed', Ethar H. A. Elsheikh', Noon H. Khalid', \\ Samia A. Mohammed', Sara O. Elmobark' , Ola O. Ali', Duaa A. Mohammed Ali', Mohamed S. Muneer', \\ Heitham Awadalla ${ }^{8}$ and Elfatih M. Malik ${ }^{8}$
}

\begin{abstract}
Background: Coronavirus disease 2019 (COVID-19) is a pandemic caused by a newly discovered coronavirus. Although clinical manifestations of COVID-19 are mainly pulmonary, some patients have other systemic manifestations. This study aimed to describe the clinical finding and outcomes in Sudanese patients diagnosed with COVID-19.

Methods: This retrospective observational study is based on documented files that included patients diagnosed with COVID-19 in seven selected hospitals inside Khartoum. Clinical manifestations, complications and outcomes were extracted from patients' records using an extraction form designed for this study.

Results: Data of 243 patients diagnosed with COVID-19 were analyzed. The mean (SD) age in years was 55.8 (18.4). Out of 116 participants, 27 of them (23.3\%) had severe disease, 15 (12.9\%) were critically ill. $67.5 \%$ of patients were admitted to the hospital within 7 days from onset of symptoms; most of them were admitted to the wards $(n=140,72.5 \%)$. Fever (83.2\%), cough (70.7\%), and shortness of breath (69.2\%) were the most commonly recorded clinical manifestations. Sepsis (9.8\%) and acidosis (7.8\%) were the most frequently reported complications. Death was the final outcome in $21.4 \%(56 / 243)$. Older age and presence of diabetes were found significantly associated with in-hospital death. The laboratory results showed high CRP in 85.6\% (119/139), high ferritin in 88.9\% (24/27), lactate dehydrogenase had a median of 409.0 (359-760), D-dimer had a median of 3.3 (1.2-16. 6), and 53/105 (50.5\%) had low albumin.
\end{abstract}

Conclusions: Fever was the most mentioned sign among the participants, followed by fatigue. Cough and shortness of breath were the most commonly recorded pulmonary symptoms manifested. Our study showed multiple variables were associated with in-hospital death. The mortality rate was high among severe and critically ill patients diagnosed with COVID-19.

Keywords: COVID-19, Clinical manifestations, In-hospital outcomes, Complications, Sudan, Severity

*Correspondence: elfatih.ahmed.hasabo@gmail.com

${ }^{1}$ Faculty of Medicine, University of Khartoum, ElQasr Avenue, Khartoum, Khartoum state 11111, Sudan

Full list of author information is available at the end of the article

\section{Introduction}

COVID-19 is an infectious disease that rapidly evolved into a pandemic caused by a newly discovered coronavirus. Coronaviruses were identified for the first time in the mid-1960s [1], and the new coronavirus strain, original author(s) and the source, provide a link to the Creative Commons licence, and indicate if changes were made. The images or other third party material in this article are included in the article's Creative Commons licence, unless indicated otherwise in a credit line to the material. If material is not included in the article's Creative Commons licence and your intended use is not permitted by statutory regulation or exceeds the permitted use, you will need to obtain permission directly from the copyright holder. To view a copy of this licence, visit http://creativecommons.org/licenses/by/4.0/. 
namely severe acute respiratory syndrome coronavirus 2 (SARS-Cov-2), is related to the cluster of acute respiratory diseases in Wuhan, China, in December 2019 [2]. There were two highly infectious human coronaviruses in the past decades: the coronavirus responsible for the severe acute respiratory syndrome (SARS-Cov) and the coronavirus responsible for the Middle East respiratory syndrome (MERS-Cov) (1). They caused lower respiratory tract infections and extra-pulmonary manifestations, leading to an increase in the mortality rates of thousands of cases up to $50 \%$ in specific populations [2]. The COVID-19 symptoms and signs in most infected people will be mild-to-moderate respiratory diseases and recover with supportive treatment (2). Older people and those with comorbidities like cardiovascular disease, chronic respiratory disease, diabetes, cancer, and other immune diseases are more likely to develop a severe form of the disease [3].

Although clinical manifestations of COVID-19 are mainly pulmonary, some patients have other systemic manifestations such as cardiovascular, neurological, hematopoietic, and immune system manifestations [2]. Besides, several data reported that COVID-19 should be considered as a multisystem disease in which patients can present with chest tightness and palpitations instead of cough and other respiratory symptoms [2]. Furthermore, it was found that lymphopenia was the prevalent hematological findings in many patients [4]. Additionally, the high C-reactive protein (CRP), serum ferritin, lactate dehydrogenase (LDH), and several other immunological markers were associated with an increased risk of COVID-19 and death [5]. Also, coagulopathy has been reported in different cases, manifested by elevated D-dimer, prothrombin time (PT), and partial thromboplastin time (PTT) [6]. The risk of acute respiratory distress syndrome (ARDS) is associated with higher D-dimer and prothrombin time [7].

Several risk factors that increase mortalities among patients diagnosed with COVID-19 have been identified, such as older age, male sex, hypertension, diabetes, and cardiovascular diseases. In addition to that, there is still uncertainty regarding the patient of potential confounding [8].

On the 13th of March 2020, Sudan reported its first novel coronavirus case in Khartoum, and up to Monday 25th of January 2021, the total number of patients diagnosed with COVID-19 reached 28,522, including 1722 associated deaths, CFR: 6.5\% [9]. Unfortunately, comprehensive knowledge of COVID-19 remains incomplete, and many essential features are still unknown. Moreover, since the emergence of COVID-19, the disease presenting symptoms were changing with time and place, and there were no previous published studies about outcomes of patients with COVID-19 in Sudan. We conducted this study to describe the clinical findings and outcomes in patients with COVID-19 in Sudan, Africa.

\section{Materials and methods Study design and population}

This retrospective observational study included patients diagnosed with COVID-19 between the 1st of April and 30th of September 2020 in 7 selected hospitals inside Khartoum State, Sudan. Khartoum State is the capital of Sudan, and it has about 20 percent of the country's total population, according to the latest estimate from the Integrated Food Security Phase Classification (IPC).

The Ministry of Health established three main isolation centers in Khartoum state, involving Khartoum teaching hospital, Jebra trauma center, and The Universal hospital. Additionally, many hospitals in public and private sectors have identified several beds for patients with COVID-19. Our study was conducted in seven hospitals in Khartoum state, which are:

1- Jebra Hospital for emergency and injuries is a governmental hospital located in Jebra, block 19, Khartoum city, with 88 beds for confirmed cases, in addition to an intensive care unit.

2- Khartoum Teaching Hospital is a public hospital existing in Isbitalia Street, Khartoum city, with 30 beds for patients with COVID-19, in addition to an Intensive care unit equipped with mechanical ventilators.

3- The Universal Hospital is an acute care tertiary facility, in Bahri city, Kober East Alwaha, with a bed capacity of 248 inpatient rooms divided into six floors.

4- Royal Care Hospital is a private, tertiary care hospital located in Mohamed Salah El-Din Street, Khartoum city, with 20 beds available for patients with COVID19 , in addition to an Intensive care unit.

5- Al Saha Specialized Hospital is a private hospital in Khartoum city, with a 20-30 bed capacity for patients with COVID-19.

6- Omdurman Teaching Hospital is a governmental hospital situated in Omdurman city, with 40 beds for patients with COVID-19.

7- Al-Baraha Medical City is a private hospital located et al.-Baraha Street, Bahri city, with 30 beds for patients with COVID-19.

We included Sudanese patients diagnosed with COVID-19 using nasopharyngeal swab tested by RTPCR, 18 years old and older, and admitted to the mentioned hospitals. We excluded all pregnant women, 
asymptomatic patients, and patients lacking information necessary for the study in their files.

Patients were classified and treated according to the COVID-19 case management protocols released by the Federal Ministry of Health of the Republic of Sudan during the epidemic [10]. The mild form of the disease was defined as COVID-19 with uncomplicated, non-specific upper respiratory tract symptoms, for instance, sore throat, nasal congestion, and cough, in addition to mild general manifestations, including fever and malaise. The moderate disease was defined as uncomplicated pneumonia with oxygen saturation $>92 \%$ on room air and radiological evidence (typically bilateral ground-glass opacities). Patients with the severe disease are typically in respiratory distress with oxygen saturation $<92$ on room air and radiological evidence of severe pneumonia. After assessing and classifying patients, those with moderate disease were admitted to isolation areas and treated empirically with amoxicillin $500 \mathrm{mg} / 8 \mathrm{~h}+$ azithromycin $500 \mathrm{mg}$ daily for 3 days, with hydroxy-chloroquine $400 \mathrm{mg} / 12 \mathrm{~h}$ for one day followed by $400 \mathrm{mg}$ for a total of 5 days. Patients with severe disease were admitted to a monitored bed with supplemental oxygen to keep saturation $>92 \%$, and ceftriaxone $1 \mathrm{~g} / 24 \mathrm{~h}+$ azithromycin $500 \mathrm{mg} / 24 \mathrm{~h}$ were prescribed, in addition to hydroxychloroquine $400 \mathrm{mg} / 12 \mathrm{~h}$ for one day, followed by $400 \mathrm{mg}$ for a total of 5 days. Antivirals and immunomodulators were preserved for specific cases. Critically ill patients were admitted to ICUs and treated as severe sepsis. Noninvasive positive pressure ventilation/endotracheal intubation and mechanical ventilation were used to manage ARDS [10].

\section{Sampling technique and data collection}

We covered all patients diagnosed with COVID-19 in our seven selected and previously mentioned hospitals, from the 1st of April to the 30th of September 2020. Data were collected from patients' records and laboratory sheets using an extraction form. The form contained the following variables: sociodemographic characteristics, the severity of the disease, comorbidities, vital signs, clinical manifestations, duration from the onset of symptom to hospital admission, admission area, in-hospital complications, length of stay, short- and long-term in-hospital outcomes, and laboratory investigations including [red blood cells (RBCs), hemoglobin, hematocrit, white blood cells (WBCs), platelets, CRP, erythrocyte sedimentation rate (ESR), ferritin, lactate dehydrogenase, random blood sugar, sodium, potassium, blood urea nitrogen, creatinine, alanine aminotransferase, aspartate aminotransferase, alkaline phosphatase, total bilirubin, albumin and D-dimer].

\section{Statistical analysis plan}

We described the data for all patients diagnosed with COVID-19 using SPSS version 25. Continuous data were presented as mean \pm standard deviation (SD) or median (interquartile range). Categorical data were presented as numbers (percentages). Test of normality was used to find the distribution of continuous variables. Spearman's correlation was used to find correlation of variables with severity of the disease or length of stay. A univariate logistic regression was used to find predictors of in-hospital death in patients with COVID19. A $P$-value less than 0.05 is considered significant.

\section{Results}

A total of 393 files were reached in this study, out of which 150 files were excluded due to incomplete necessary information (age, gender, comorbidities, presentation, complications, and the outcome). Accordingly, 243 files were included in this study.

\section{Sociodemographic data of patients with COVID-19}

The mean age of the patients was $55.8 \pm 18.4$ years, and just over half of them $(n=126,51.9 \%)$ were 60 years or above. More than half of the participants were males $(n=145,59.7 \%)$ (Table 1).

\section{Comorbidities}

Hypertension and diabetes were the most commonly recorded comorbidities in $(n=85,35 \%)$ and $(n=75$, $30.9 \%$ ) of patients, respectively (Table 1 ).

\section{In-hospital characteristics of patients with COVID-19}

Respiratory manifestations were the most common clinical features among patients diagnosed with COVID-19 $(n=198,81.5 \%)$, and a large proportion of them had a cough $(n=140,70.7 \%)$. Fever was the leading complaint in the vast majority of patients $(n=144$, 83.2\%). Also, gastrointestinal features were presented in a small minority of patients $(n=42,17.3 \%)$, and diarrhea was the most common feature $(n=18,42.9 \%)$. Furthermore, only a mere $9 \%$ of patients complained of neurological symptoms $(n=23,9.5 \%)$, and in the vast majority, a disturbing level of consciousness was the main complaint $(n=21,91.3 \%)$ (Table 2 ).

Most of the patients reached the hospital within 7 days from the onset of symptoms $(n=114 / 169$, $67.5 \%$ ), and nearly half of them stayed at the hospital for 7 days or less $(n=98 / 201,48.8 \%)$. A significant 
Table 1 Sociodemographic characteristics and comorbidities of patients with COVID-19 and their association with the final outcome $(N=243)$

\begin{tabular}{|c|c|c|c|c|c|}
\hline \multirow[t]{2}{*}{ Variables } & \multirow[t]{2}{*}{$N(\%)$ or mean $\pm S D$} & \multicolumn{4}{|c|}{ Univariate logistic regression for predictors of in-hospital death } \\
\hline & & OR & Lower $\mathrm{Cl}$ & Upper Cl & P-value \\
\hline Age, years, mean $\pm S D$ & $55.8 \pm 18.4$ & 1.021 & 1.002 & 1.039 & 0.026 \\
\hline \multicolumn{6}{|l|}{ Age groups (years) } \\
\hline $18-29$ & $34(14.0)$ & & & & \\
\hline $30-39$ & $22(9.1)$ & & & & \\
\hline $40-49$ & $24(9.9)$ & & & & \\
\hline $50-59$ & $37(15.2)$ & & & & \\
\hline $60-70$ & $69(28.4)$ & & & & \\
\hline$>70$ & $57(23.5)$ & & & & \\
\hline \multicolumn{6}{|l|}{ Gender } \\
\hline Male (reference) & $145(59.7)$ & - & - & - & - \\
\hline Female & $98(40.3)$ & 0.905 & 0.483 & 1.698 & 0.757 \\
\hline \multicolumn{6}{|l|}{ Comorbidities } \\
\hline Hypertension & $85(35.0)$ & 0.701 & 0.359 & 1.367 & 0.297 \\
\hline Diabetes & $75(30.9)$ & 1.909 & 1.012 & 3.602 & 0.046 \\
\hline Cardiovascular disease & $17(7.0)$ & 1.587 & 0.533 & 4.727 & 0.407 \\
\hline Asthma & $16(6.6)$ & 0.23 & 0.03 & 1.784 & 0.16 \\
\hline Smoking & $19(7.8)$ & 0.67 & 0.187 & 2.392 & 0.537 \\
\hline Chronic kidney disease & $18(7.4)$ & 0.438 & 0.097 & 1.967 & 0.281 \\
\hline Cerebrovascular accident & $9(3.7)$ & 3.1 & 0.802 & 11.988 & 0.101 \\
\hline Liver disease & $1(0.4)$ & - & - & - & 1 \\
\hline Pulmonary tuberculosis & $1(0.4)$ & - & - & - & 1 \\
\hline Alcohol & $1(0.4)$ & - & - & - & 1 \\
\hline
\end{tabular}

majority of the participants were admitted to wards $(n=140 / 193,72.5 \%)$ (Table 2).

\section{Laboratory investigations}

Regarding vital signs, patients had a median heart rate of 90 (81-103) beats per minute and median respiratory rate of 24 (20-36) breath per minute. Most of them were within the hypertensive systolic range (129/196, $65.8 \%)$ and hypertensive diastolic range (106/196, 54.1\%) (Table 3).

Regarding laboratory tests, though most of patients had normal WBCs and neutrophil counts (102/164, $62.2 \%)$ and $(79 / 168,57.2 \%)$, respectively, most of them had lymphopenia $(85 / 145,58.6 \%)$. Other important findings include elevated CRP (98/112, 87.5\%), high ferritin level $(21 / 22,95.5 \%)$ and hypoalbuminemia (44/88, 50\%) (Table 3).

\section{In-hospital complications, severity, interventions, and outcomes of COVID-19 Complications}

Although, three-quarters of patients did not develop any complications $(n=173 / 243, \quad 71.2 \%)$, sepsis $(n=24 / 243,9.8 \%)$ and acidosis $(n=19 / 243,7.8 \%)$ were the most commonly mentioned complications (Table 2).

\section{Severity of the disease}

Out of 243 patients enrolled, only 116 were classified in the documents according to the disease severity score. $38 \%$ of those patients had the mild disease $(n=45$, $38.8 \%)$, and precisely, a quarter of them had moderate disease $(29,25.0 \%)$. Additionally, severe disease was found in $(n=27,23.3 \%)$, and a few of the patients were critically ill $(15,12.9 \%)$ (Table 2$)$.

\section{Interventions}

Concerning the need for respiratory support, nearly a fifth of the participants $(n=47 / 182,25.8 \%)$ needed this intervention, in which more than half of them required non-invasive ventilation $(n=25,53.2 \%)$, compared to $(n=13,27.7 \%)$ who needed invasive ventilation. In the remaining files $(n=9,19.1 \%)$, the type of ventilation was not recorded (Table 4 ). 
Table 2 Clinical manifestations: symptoms, hospital disposition, complications and severity of patients diagnosed with COVID-19 and their association with the final outcome

\begin{tabular}{|c|c|c|c|c|c|c|}
\hline \multirow[t]{2}{*}{ Variables } & \multirow[t]{2}{*}{$n$} & \multirow[t]{2}{*}{$\begin{array}{l}\text { Number (\%) or } \\
\text { median (IQR) }\end{array}$} & \multicolumn{4}{|c|}{$\begin{array}{l}\text { Univariate logistic regression for predictors of in-hospita } \\
\text { death }\end{array}$} \\
\hline & & & OR & Lower $\mathrm{Cl}$ & Upper Cl & $P$-value \\
\hline $\begin{array}{l}\text { Duration from the onset of symptom to hospital } \\
\text { admission, days }\end{array}$ & 169 & $5(3-10)$ & 1.002 & 0.939 & 1.07 & 0.943 \\
\hline 7 days and less & & $114(67.5)$ & & & & \\
\hline 8-14 days & & $44(26.0)$ & & & & \\
\hline $15-30$ days & & $11(6.5)$ & & & & \\
\hline More than 30 days & & $0(0.0)$ & & & & \\
\hline Site of disposition at admission & 193 & & & & & \\
\hline Ward deposition (reference) & & $140(72.5)$ & - & - & - & - \\
\hline HDU deposition ${ }^{\mathrm{a}}$ & & $50(25.9)$ & 5.231 & 2.538 & 10.781 & $<0.001$ \\
\hline ICU deposition ${ }^{\mathrm{a}}$ & & $3(1.6)$ & 2.833 & 0.246 & 32.666 & 0.404 \\
\hline \multicolumn{7}{|l|}{ Manifestations } \\
\hline Pulmonary manifestations & 198 & & & & & \\
\hline Cough & & $140(70.7)$ & 0.627 & 0.308 & 1.279 & 0.2 \\
\hline Shortness of breath & & $137(69.2)$ & 3.392 & 1.347 & 8.538 & 0.010 \\
\hline Hemoptysis & & $5(2.5)$ & 0.899 & 0.098 & 8.258 & 0.925 \\
\hline Chest pain & & $12(6.1)$ & - & - & - & 0.999 \\
\hline Sore throat & & $32(16.2)$ & 0.326 & 0.094 & 1.127 & 0.077 \\
\hline Congestion/runny nose & & $9(4.5)$ & - & - & - & 0.999 \\
\hline General manifestations & 173 & & & & & \\
\hline Fever & & $144(83.2)$ & 1.263 & 0.445 & 3.588 & 0.661 \\
\hline Fatigue & & $58(33.5)$ & 0.887 & 0.4 & 1.967 & 0.796 \\
\hline Headache & & $40(23.1)$ & 0.159 & 0.036 & 0.697 & 0.015 \\
\hline Body aches & & $17(9.8)$ & 1.241 & 0.378 & 4.069 & 0.722 \\
\hline Loss of smell & & $4(2.3)$ & - & - & - & 0.999 \\
\hline Loss of taste & & $1(0.6)$ & - & - & - & 1 \\
\hline Neurological manifestations & 23 & & & & & \\
\hline Disturbed level of consciousness & & $21(91.3)$ & - & - & - & 0.999 \\
\hline Hemiparesis & & $4(17.4)$ & 1.111 & 0.129 & 9.605 & 0.924 \\
\hline Aphasia & & $3(13.0)$ & 2.444 & 0.19 & 31.526 & 0.493 \\
\hline Hemi sensory deficit & & $0(0.0)$ & - & - & - & - \\
\hline Visual loss & & $0(0.0)$ & - & - & - & - \\
\hline Gastrointestinal manifestations & 42 & & & & & \\
\hline Nausea/vomiting & & $15(35.7)$ & 2.933 & 0.711 & 12.108 & 0.137 \\
\hline Diarrhea & & $18(42.9)$ & 1.154 & 0.289 & 4.608 & 0.839 \\
\hline Abdominal pain & & $13(30.9)$ & 0.788 & 0.171 & 3.621 & 0.759 \\
\hline Loss of appetite & & $8(19.0)$ & 0.343 & 0.037 & 3.161 & 0.345 \\
\hline Vascular manifestation & 3 & & & & & \\
\hline $\mathrm{DVT}^{\mathrm{a}}$ & & $2(66.7)$ & - & - & - & 1 \\
\hline Coronary artery disease & & $1(33.3)$ & - & - & - & 1 \\
\hline Bowel ischemia & & $0(0.0)$ & - & - & - & - \\
\hline Limb ischemia & & $0(0.0)$ & - & - & - & - \\
\hline In-hospital complications (yes) & 243 & $70(28.8)$ & & & & \\
\hline Sepsis & & $24(9.8)$ & 15.074 & 5.751 & 39.511 & $<0.001$ \\
\hline Acidosis & & $19(7.8)$ & 32.711 & 8.986 & 119.081 & $<0.001$ \\
\hline Respiratory failure & & $17(6.9)$ & 44.758 & 9.753 & 205.392 & $<0.001$ \\
\hline Acute kidney injury & & $15(6.2)$ & 21.647 & 5.800 & 80.791 & $<0.001$ \\
\hline Acute respiratory distress syndrome & & $12(4.9)$ & 6.533 & 1.971 & 21.661 & 0.002 \\
\hline
\end{tabular}


Table 2 (continued)

\begin{tabular}{|c|c|c|c|c|c|c|}
\hline \multirow[t]{2}{*}{ Variables } & \multirow[t]{2}{*}{$n$} & \multirow[t]{2}{*}{$\begin{array}{l}\text { Number (\%) or } \\
\text { median (IQR) }\end{array}$} & \multicolumn{4}{|c|}{$\begin{array}{l}\text { Univariate logistic regression for predictors of in-hospital } \\
\text { death }\end{array}$} \\
\hline & & & OR & Lower $\mathrm{Cl}$ & Upper Cl & $P$-value \\
\hline Hyponatremia & & $10(4.1)$ & 0.44 & 0.054 & 3.559 & 0.441 \\
\hline Alkalosis & & $8(3.3)$ & 7.480 & 1.718 & 32.558 & 0.007 \\
\hline Hypokalemia & & $8(3.3)$ & 1.371 & 0.268 & 7.026 & 0.705 \\
\hline Shock & & $6(2.5)$ & - & - & - & 0.999 \\
\hline Hyperkalemia & & $5(2.1)$ & - & - & - & 0.999 \\
\hline Acute cardiac injury & & $4(1.6)$ & 4.205 & 0.576 & 30.677 & 0.157 \\
\hline Heart failure & & $2(0.8$ & - & 0 & & 0.999 \\
\hline GIT bleeding ${ }^{\mathrm{a}}$ & & $2(0.8)$ & & 0 & & 0.999 \\
\hline Hypernatremia & & $1(0.4)$ & - & - & - & 1 \\
\hline Acute liver injury & & $0(0.0)$ & - & - & - & - \\
\hline Disseminated intravascular coagulation & & $0(0.0)$ & - & - & - & - \\
\hline Severity of the disease & 116 & & & & & \\
\hline Mild (reference) & & $45(38.8)$ & - & - & - & - \\
\hline Moderate & & $29(25.0)$ & 3.259 & 0.282 & 37.687 & 0.344 \\
\hline Severe & & $27(23.3)$ & 40.857 & 4.899 & 340.728 & 0.001 \\
\hline Critically ill & & $15(12.9)$ & 286.000 & 23.977 & 3411.477 & $<0.001$ \\
\hline
\end{tabular}

Bold values mean these varibles were statistically significant

${ }^{a} \mathrm{HDU}$, high dependency unit; ICU, intensive care unit; DVT, deep vein thrombosis; GIT, gastrointestinal tract

\section{Outcomes}

$n=131 / 193,67.9 \%$ of participants were discharged home within 15 days of their admission and $n=191 / 243,78.6 \%$ discharged in the long-term (Table 4).

Factors influencing the outcome in patients with COVID-19 In univariate logistic regression, some factors were found significantly associated and predict the final outcome (inhospital death). These factors were: increase in age (OR: 1.021 , CI 95\% 1.002-1.039; $P=0.026$ ), presence of diabetes (OR: 1.909, CI 95\% 1.012-3.602; $P=0.046$ ), deposition to HDU at the time of admission (OR: 5.231, CI 95\% 2.538-10.781; $\left.P^{<} 0.001\right)$, admission with shortness of breath (OR: 3.392, CI 95\% 1.347-8.538; $P=0.010$ ), absence of headache at the time of admission (OR: 0.159, CI 95\% 0.036-0.697; $P=0.015$ ), sepsis (OR: 15.074, CI 95\% 5.751-39.511; $P^{<} 0.001$ ), acidosis (OR: 32.711, CI 95\% 8.986-119.081; $P^{<} 0.001$ ), respiratory failure (OR: 44.758, CI 95\% 9.753-205.392; $\left.P^{<} 0.001\right)$, acute kidney injury (OR: 21.647, CI 95\% 5.800-80.791; $P^{<} 0.001$ ), acute respiratory distress syndrome (OR: 6.533, CI 95\% 1.971-21.661; $P=0.002$ ), alkalosis (OR: 7.480, CI 95\% 1.718-32.558; $P=0.007)$, patient with severe disease (OR: 40.857, CI 95\% 4.899-340.728; $P=0.001$ ) and patient with critically ill disease (OR: 286.000, CI 95\% 23.977-3411.477; $\left.P^{<} 0.001\right)$. The predictors of in-hospital death in laboratory investigations and vital signs were: increase in heart rate (OR: 1.052, CI 95\% 1.030-1.076;
$P^{<} 0.001$ ), increase in respiratory rate (OR: $1.061, \mathrm{CI} 95 \%$ 1.022-1.103; $P=0.002$ ), decrease in $\mathrm{SpO}^{2}$ (OR: $0.903, \mathrm{CI}$ 95\% 0.866-0.942; $P^{<} 0.001$ ), low RBCs counts (OR: 0.903, CI 95\% 0.866-0.942; $P=0.024)$, low hemoglobin concentration (OR: 0.765 , CI 95\% 0.651-0.899; P=0.001), low hematocrit (OR: 0.894, CI 95\% 0.836-0.956; $P=0.001$ ), high WBCs (OR: 1.144, CI 95\% 1.074-1.218; $P^{<} 0.001$ ), high neutrophils (OR: 1.125 , CI 95\% 1.049-1.205; $P=0.001$ ), high CRP (OR: 1.006, CI 95\% 1.002-1.010; $P=0.003$ ), high random blood sugar (OR: 1.004 , CI $95 \%$ 1.001-1.008; $P=0.024$ ), high potassium (OR: $1.742, \mathrm{CI}$ 95\% 1.093-2.777; $P=0.020$ ), high blood urea nitrogen (OR: 1.010, CI 95\% 1.004-1.018; $P=0.003$ ), high alanine aminotransferase (OR: 1.014, CI 95\% 1.002-1.025; $P=0.022$ ), high aspartate aminotransferase (OR: 1.018, CI 95\% 1.002-1.035; $P=0.024$ ), high total bilirubin (OR: 3.426, CI 95\% 1.087-10.803; $P=0.036)$ and usage of respiratory support (OR: 16.612, CI 95\% 7.113-38.800; $P^{<} 0.001$ ) (Tables 1, 2, 3, 4).

\section{Factors associated with COVID-19 severity}

Several factors were found to be significantly positively correlated with the disease severity. These factors includes: Age ( $p$-value $\leq 0.001, \quad R=0.497)$, diabetes $\quad(p$-value $=0.016, \quad R=0.224), \quad$ hypertension $(p$-value $\leq 0.001, R=0.373)$, cardiovascular disease $\quad(p$-value $=0.002, \quad R=0.288), \quad$ cerebrovascular disease $(p$-value $=0.005, R=0.258)$, shortness of breath 
Table 3 Vital signs and laboratory investigations among patients diagnosed with COVID-19 and their association with the final outcome

\begin{tabular}{|c|c|c|c|c|c|c|}
\hline \multirow[t]{2}{*}{ Variables } & \multirow[t]{2}{*}{$n$} & \multirow[t]{2}{*}{ Number (\%) or median (IQR) } & \multicolumn{4}{|c|}{$\begin{array}{l}\text { Univariate logistic regression for predictors of } \\
\text { in-hospital death }\end{array}$} \\
\hline & & & $\overline{\mathrm{OR}}$ & Lower Cl & Upper Cl & $P$-value \\
\hline \multicolumn{7}{|l|}{ Vital signs } \\
\hline Heart rate, beat/min & 189 & $90(81-103)$ & 1.052 & 1.030 & 1.076 & $<0.001$ \\
\hline Systolic BPa ${ }^{\mathrm{a} m H g}$ & 196 & $131(118.3-145)$ & 0.985 & 0.969 & 1.001 & 0.067 \\
\hline Normal & & $59(30.1)$ & & & & \\
\hline Hypertension & & $129(65.8)$ & & & & \\
\hline Hypertensive crisis & & $8(4.1)$ & & & & \\
\hline Diastolic $\mathrm{BP}^{\mathrm{a}}, \mathrm{mmHg}$ & 196 & $80(70.25-90)$ & 1.001 & 0.999 & 1.002 & 0.47 \\
\hline Normal & & $85(43.4)$ & & & & \\
\hline Hypertension & & $106(54.1)$ & & & & \\
\hline Hypertensive crisis & & $5(2.6)$ & & & & \\
\hline Respiratory rate, breath/min & 116 & $24(20-36)$ & 1.061 & 1.022 & 1.103 & 0.002 \\
\hline Temperature, centigrade & 93 & $37(36.5-37.8)$ & 0.956 & 0.776 & 1.178 & 0.674 \\
\hline SpO ${ }^{2 a}$, percentage & 182 & 95 (89-98) & 0.903 & 0.866 & 0.942 & $<0.001$ \\
\hline \multicolumn{7}{|l|}{ Laboratory Investigation } \\
\hline Red blood cells counts, million $/ \mathrm{mm}^{3}$ & 128 & $4.3(3.6-4.9)$ & 0.566 & 0.345 & 0.928 & 0.024 \\
\hline Hemoglobin, g/dL & 161 & $11.9(10.1-13.6)$ & 0.765 & 0.651 & 0.899 & 0.001 \\
\hline Hematocrit, percentage & 129 & $36(31-41)$ & 0.894 & 0.836 & 0.956 & 0.001 \\
\hline WBC s counts ${ }^{\mathrm{a}}, \times 103 / \mu \mathrm{L}$ & 164 & $7.3(5,2-12.1)$ & 1.144 & 1.074 & 1.218 & $<0.001$ \\
\hline Leukopenia & 164 & $15(9.1)$ & & & & \\
\hline Normal & & $102(62.2)$ & & & & \\
\hline Leukocytosis & & $47(28.7)$ & & & & \\
\hline Monocyte, $\times 10^{3} / \mu \mathrm{L}$ & 103 & $0.5(0.4-0.8)$ & 1.624 & 0.854 & 3.087 & 0.139 \\
\hline Lymphocyte, $\times 10^{3} / \mu \mathrm{L}$ & 145 & $1.3(0.8-2.0)$ & 1.014 & 0.776 & 1.326 & 0.919 \\
\hline Lymphopenia & 145 & $85(58.6)$ & & & & \\
\hline Normal & & $59(40.7)$ & & & & \\
\hline Lymphocytosis & & $1(0.7)$ & & & & \\
\hline Neutrophils, $\times 10^{3} / \mu \mathrm{L}$ & 168 & 5.25 (3.19-9.8) & 1.125 & 1.049 & 1.205 & 0.001 \\
\hline Neutropenia & 168 & $11(8.0)$ & & & & \\
\hline Normal & & $79(57.2)$ & & & & \\
\hline Neutrophilia & & $48(34.8)$ & & & & \\
\hline Platelets, $\times 10^{9} / \mathrm{L}$ & 87 & $247.5(187.5-342.3)$ & 0.997 & 0.994 & 1.001 & 0.101 \\
\hline $\mathrm{CRP}, \mathrm{mg} / \mathrm{dl}^{\mathrm{a}}$ & 112 & $62(18.1-143.8)$ & 1.006 & 1.002 & 1.010 & 0.003 \\
\hline Normal CRP level & & $14(12.5)$ & & & & \\
\hline High CRP level & & $98(87.5)$ & & & & \\
\hline ESR, mm/hour & 64 & $40(15-68)$ & 1.018 & 0.998 & 1.039 & 0.078 \\
\hline Ferritin, ng/ml & 22 & $651.9(470.4-1472.8)$ & 1 & 0.999 & 1.001 & 0.658 \\
\hline Normal Ferritin level & & $1(4.5)$ & & & & \\
\hline High Ferritin level & & $21(95.5)$ & & & & \\
\hline Lactate dehydrogenase, unit/liter & 19 & $409.0(359-760)$ & 1 & 0.998 & 1.001 & 0.688 \\
\hline Random blood sugar, mg/dL & 112 & $141(102.5-261.8)$ & 1.004 & 1.001 & 1.008 & 0.024 \\
\hline Sodium, mmol/L & 144 & $135(130-139)$ & 1.04 & 0.994 & 1.088 & 0.086 \\
\hline Potassium, mmol/L & 146 & $3.8(3.5-4.3)$ & 1.742 & 1.093 & 2.777 & 0.020 \\
\hline Blood Urea nitrogen, mg/dL & 149 & $30(17-50.5)$ & 1.010 & 1.004 & 1.018 & 0.003 \\
\hline Creatinine, $\mathrm{mg} / \mathrm{dL}$ & 151 & $1(0.7-2.2)$ & 1.003 & 0.992 & 1.014 & 0.585 \\
\hline Alanine aminotransferase, unit /liter & 78 & $26(19-43.7)$ & 1.014 & 1.002 & 1.025 & 0.022 \\
\hline Aspartate aminotransferase, unit /liter & 73 & $28(17-56.7)$ & 1.018 & 1.002 & 1.035 & 0.024 \\
\hline
\end{tabular}


Table 3 (continued)

\begin{tabular}{|c|c|c|c|c|c|c|}
\hline \multirow[t]{2}{*}{ Variables } & \multirow[t]{2}{*}{$n$} & \multirow[t]{2}{*}{ Number (\%) or median (IQR) } & \multicolumn{4}{|c|}{$\begin{array}{l}\text { Univariate logistic regression for predictors of } \\
\text { in-hospital death }\end{array}$} \\
\hline & & & OR & Lower Cl & Upper Cl & $P$-value \\
\hline Alkaline phosphatase, unit /liter & 70 & $96(63.5-119)$ & 1.001 & 0.998 & 1.003 & 0.55 \\
\hline Total bilirubin, mg/dl & 55 & $0.6(0.3-0.9)$ & 3.426 & 1.087 & 10.803 & 0.036 \\
\hline Albumin, g/dl & 88 & $3.5(2.9-4.2)$ & 1.016 & 0.985 & 1.048 & 0.312 \\
\hline Low Albumin level & & $44(50.0)$ & & & & \\
\hline Normal Albumin level & & $41(46.6)$ & & & & \\
\hline High Albumin level & & $3(3.4)$ & & & & \\
\hline D-dimer & 26 & $3.3(1.2-16.6)$ & 0.939 & 0.804 & 1.097 & 0.429 \\
\hline
\end{tabular}

Bold values mean these varibles were statistically significant

${ }^{a} \mathrm{BP}$, blood pressure; $\mathrm{SpO}^{2}$, saturation of peripheral oxygen; WBCs, white blood cells; CRP, C-reactive protein; ESR, estimation sedimentation rate

Table 4 Respiratory support and outcomes of patients diagnosed with COVID-19 and the association of respiratory support with final outcome

\begin{tabular}{|c|c|c|c|c|c|c|}
\hline \multirow[t]{2}{*}{ Variables } & \multirow[t]{2}{*}{$n$} & \multirow{2}{*}{$\begin{array}{l}\text { Number (\%) or } \\
\text { median (IQR) }\end{array}$} & \multicolumn{4}{|c|}{ Univariate logistic regression } \\
\hline & & & $\overline{\text { OR }}$ & Lower $\mathrm{Cl}$ & Upper Cl & $P$-value \\
\hline Respiratory support & 182 & & & & & \\
\hline Not used & & $135(74.2)$ & - & - & - & - \\
\hline Used & & $47(25.8)$ & 16.612 & 7.113 & 38.800 & $<0.001$ \\
\hline Type of respiratory support used & 38 & & & & & \\
\hline Non-invasive & & $25(65.8)$ & & & & \\
\hline Invasive & & $13(34.2)$ & & & & \\
\hline Short term outcome (after 15 days) & 193 & & & & & \\
\hline Discharge home & & $131(67.9)$ & & & & \\
\hline Death & & $34(17.6)$ & & & & \\
\hline HDU transfer ${ }^{a}$ & & $5(2.6)$ & & & & \\
\hline ICU transfer ${ }^{\mathrm{a}}$ & & $19(9.8)$ & & & & \\
\hline Others & & $4(2.1)$ & & & & \\
\hline Outcome & 243 & & & & & \\
\hline Discharge home & & $191(78.6)$ & & & & \\
\hline Death & & $52(21.4)$ & & & & \\
\hline $\begin{array}{l}\text { In-hospital stay from admission to the } \\
\text { outcome, Days }\end{array}$ & 201 & $8(4-14.5)$ & & & & \\
\hline 7 days and less & & $98(48.8)$ & & & & \\
\hline $8-14$ days & & $53(26.4)$ & & & & \\
\hline $15-30$ & & $42(20.9)$ & & & & \\
\hline More than 30 & & $8(3.9)$ & & & & \\
\hline
\end{tabular}

Bold values mean these varibles were statistically significant

${ }^{a}$ HDU, High Dependency Unit; ICU, Intensive Care Unit

( $p$-value $\leq 0.001, R=0.479)$, acute respiratory distress syndrome $(p$-value $=0.015, R=0.233)$, respiratory failure ( $p$-value $\leq 0.001, R=0.352)$, sepsis ( $p$-value $=0.002$, $R=0.292), \quad$ acidosis $\quad(p$-value $\leq 0.001, \quad R=0.400)$, alkalosis $\quad(p$-value $=0.002, \quad R=0.292), \quad$ acute kidney injury ( $p$-value $=0.017, R=0.230)$, hypokalemia $(p$-value $=0.025, \quad R=0.215), \quad$ shock $\quad(p$-value $=0.031$, $R=0.208)$, heart rate $(p$-value $=0.003, R=0.276)$, respiratory rate $(p$-value $<0.001, R=0.544), \mathrm{WBCs}$ count $(p$-value $\leq 0.001, R=0.551)$, neutrophil counts $(p$-value $\leq 0.001, R=0.651)$, CRP level $(p$-value $\leq 0.001$, $R=0.718), \quad$ ESR level $(p$-value $=0.004, \quad R=0.419)$, random blood sugar $(p$-value $\leq 0.001, \quad R=0.441)$, Sodium $\quad(p$-value $=0.005, \quad R=0.321), \quad$ blood Urea level $\quad(p$-value $\leq 0.001, \quad R=0.435), \quad$ Creatinine level $(p$-value $=0.006, R=0.293)$, alanine aminotransferase 
level ( $p$-value $=0.002, R=0.427$ ), aspartate aminotransferase level ( $p$-value $\leq 0.001, R=0.650)$, alkaline aminotransferase ( $p$-value $\leq 0.001, R=0.588)$, and total bilirubin level ( $p$-value $=0.047, R=0.349$ ).

While on the other hand factors that were found to be significantly negatively correlated with the disease severity includes: smoking ( $p$-value $\leq 0.001$, $R-0.325)$, headache $(p$-value $\leq 0.001, \quad R=-0.483)$, body aches $(p$-value $=0.025, \quad R=-0.237), \quad$ sore throat $(p$-value $\leq 0.001, \quad R=-0.357)$, runny nose ( $p$-value $=0.027, R=-0.222)$, using respiratory support ( $p$-value $\leq 0.001, R=-0.589$ ), diastolic blood pressure ( $p$-value $\leq 0.001, R=-0.354), \mathrm{SaPO} 2(p$-value $\leq 0.001$, $R=-0.730)$, lymphocyte counts ( $p$-value $\leq 0.001$, $R=-0.439)$, RBCs counts $(p$-value $=-0.511)$, hemoglobin level ( $p$-value $\leq 0.001, R=-0.496)$, hematocrit level ( $p$-value $\leq 0.001, R=-0.542$ ), and $\mathrm{D}$-dimer $(p$-value $\leq 0.001, R=-0.826)$ (Tables $4,5,6,7)$.

\section{Factors associated with length of in-hospital stay}

Several factors were found to be significantly correlated with the length of in-hospital stay, these factors are: cerebrovascular disease ( $p$-value $0.044, R=-0.142$ ), disturbed level of consciousness $(p$-value $=0.041$, $R=-0.145)$, shortness of breath ( $p$-value $=0.015$, $R=0.171)$, shortness of breath ( $p$-value $=0.044$, $R=-0.159)$, acute respiratory distress syndrome ( $p$-value $=0.019, R=0.165)$ and Acidosis ( $p$-value 0.006, $R=-0.192$ ) (Tables $4,5,6,7)$.

Table 5 Correlation between participant's characteristics and comorbidities with length of stay and severity of the disease

\begin{tabular}{|c|c|c|c|c|}
\hline \multirow[t]{3}{*}{ Variables } & \multicolumn{4}{|c|}{ Correlation } \\
\hline & \multicolumn{2}{|c|}{$\begin{array}{l}\text { Length of } \\
\text { in-hospital stay }\end{array}$} & \multicolumn{2}{|c|}{$\begin{array}{l}\text { Severity of the } \\
\text { disease }\end{array}$} \\
\hline & $R$ & $P$-value & $R$ & $P$-value \\
\hline Age & -0.012 & 0.866 & 0.497 & $<0.001$ \\
\hline Gender & -0.039 & 0.581 & -0.078 & 0.407 \\
\hline \multicolumn{5}{|l|}{ Comorbidities } \\
\hline Diabetes & -0.052 & 0.46 & 0.224 & 0.016 \\
\hline Hypertension & 0.059 & 0.407 & 0.373 & $<0.001$ \\
\hline Asthma & -0.083 & 0.243 & -0.069 & 0.485 \\
\hline Pulmonary TB & 0.036 & 0.612 & 0.004 & 0.963 \\
\hline Cardiovascular disease & 0.081 & 0.254 & 0.288 & 0.002 \\
\hline Chronic kidney disease & -0.071 & 0.317 & 0.147 & 0.109 \\
\hline Chronic liver disease & 0.002 & 0.979 & 0.147 & 0.115 \\
\hline Cerebrovascular disease & -0.142 & 0.044 & 0.258 & 0.005 \\
\hline Smoking & -0.09 & 0.206 & -0.325 & $<0.001$ \\
\hline Alcohol & -0.035 & 0.618 & - & - \\
\hline
\end{tabular}

Bold values mean these varibles were statistically significant

\section{Discussion}

This study is an observational, retrospective study to describe the clinical characteristics, complications, and outcomes of patients with COVID-19, conducted in the isolation centers of Khartoum Sudan, in April 2020, with 243 patients enrolled. We found that men are more susceptible to SARS-COV-2 (59.7\%) than women (40.3\%), which is similar to other studies conducted in Wuhan, China (73\%) [11] and New York City (60\%) [12], this may be due to several factors, including genetic factors such as the high expression of coronavirus receptors (ACE2) in men $[13,13,14]$ or behavioral characteristics. A study conducted in Spain revealed that women have a greater sense of responsibility towards the COVID-19 pandemic than men [15].

Our results revealed that the most vulnerable age groups are the elderly, especially those aged between $60-70$ years $(28.4 \%)$, followed by the age group above 70 years $(23.5 \%)$ as a result of weak immune functions [16].

In concrete with other studies [12, 17], our study revealed that hypertension is the most common underlying comorbidity among the studied cases (35\%). At the same time, diabetes mellitus, cardiovascular diseases, and asthma were reported in $30.9 \%, 7 \%$, and $6.6 \%$ of patients, respectively, and no HIV seropositive patients were identified. Moreover, we found that hypertension is the most chronic disease that can increase the severity of COVID19, with ACE2 becomes the likely explanation [18], followed by cerebrovascular and cardiovascular diseases.

When it comes to laboratory tests, leukocytosis, neutrophilia, and lymphopenia were found in (28.7\%) (34.8\%) and $(58.6 \%)$ of participants, respectively. This result is consistent with other studies conducted in China [19] and United States [20]. Also, elevated CRP and hypoalbuminemia were identified in $87.5 \%$ and $50 \%$ of patients. Interestingly, we noticed a positive correlation between these parameters and the severity of COVID 19; using them may help to identify patients with severe disease and their need for hospitalization. [ $(p=0.001)$ $(p \leq 0.0001)$, respectively].

The most common pulmonary symptoms were cough (70.7\%), followed by shortness of breath (69\%), sore throat (16.2\%), and hemoptysis (2.5\%). The development of these symptoms can be explained by the presence of severe pneumonia in patients with COVID-19. Furthermore, the severity of the disease at the time of presentation may result in such variation of the symptoms [21-23].

Although COVID-19 primarily presents with pulmonary manifestations, extra-pulmonary features have been mentioned with varying degrees of severity and 
Table 6 Correlation between clinical manifestations and complications with length of stay and severity of the disease

\begin{tabular}{|c|c|c|c|c|}
\hline \multirow[t]{3}{*}{ Variables } & \multicolumn{4}{|c|}{ Correlation } \\
\hline & \multicolumn{2}{|c|}{ Length of in-hospital stay } & \multicolumn{2}{|c|}{ Severity of the disease } \\
\hline & $R$ & $P$-value & $R$ & $P$-value \\
\hline Duration from Onset of symptom to admission & 0.015 & 0.859 & 0.187 & 0.071 \\
\hline \multicolumn{5}{|l|}{ General manifestations } \\
\hline Fever & 0.066 & 0.353 & 0.078 & 0.469 \\
\hline Headache & -0.058 & 0.411 & -0.483 & $<0.001$ \\
\hline Fatigue & 0.01 & 0.891 & -0.067 & 0.53 \\
\hline Loss of the smell sensation & 0.032 & 0.656 & -0.127 & 0.234 \\
\hline Loss of taste & -0.02 & 0.777 & -0.127 & 0.234 \\
\hline Body aches & -0.003 & 0.968 & -0.237 & 0.025 \\
\hline \multicolumn{5}{|l|}{ Neurological manifestations } \\
\hline Disturbed level of consciousness & -0.145 & 0.041 & -0.166 & 0.647 \\
\hline Hemiparesis & -0.008 & 0.913 & 0.248 & 0.489 \\
\hline Aphasia & -0.076 & 0.285 & -0.58 & 0.079 \\
\hline \multicolumn{5}{|l|}{ Pulmonary manifestations } \\
\hline Cough & -0.115 & 0.105 & 0.107 & 0.29 \\
\hline Shortness of breath & 0.171 & 0.015 & 0.479 & $<0.001$ \\
\hline Sore throat & -0.159 & 0.044 & -0.357 & $<0.001$ \\
\hline Runny nose & 0.051 & 0.471 & -0.222 & 0.027 \\
\hline Chest Pain & 0.107 & 0.129 & -0.034 & 0.753 \\
\hline Hemoptysis & 0.032 & 0.652 & 0.135 & 0.182 \\
\hline \multicolumn{5}{|l|}{ Gastrointestinal manifestations } \\
\hline Nausea and vomiting & -0.035 & 0.626 & 0.325 & 0.13 \\
\hline Diarrhea & -0.059 & 0.407 & -0.095 & 0.665 \\
\hline Abdominal pain & 0.02 & 0.778 & -0.029 & 0.897 \\
\hline Loss of appetite & -0.69 & 0.69 & -0.02 & 0.927 \\
\hline \multicolumn{5}{|l|}{ Vascular manifestations } \\
\hline DVT & -0.109 & 0.125 & -0.5 & 0.667 \\
\hline Coronary artery disease & -0.119 & 0.092 & 0.5 & 0.667 \\
\hline \multicolumn{5}{|l|}{ In-hospital complications } \\
\hline Acute respiratory distress syndrome & 0.165 & 0.019 & 0.233 & 0.015 \\
\hline Respiratory failure & -0.136 & 0.054 & 0.352 & $<0.001$ \\
\hline Acute cardiac injury & 0.015 & 0.829 & - & - \\
\hline Heart failure & -0.024 & 0.737 & 0.153 & 0.113 \\
\hline Sepsis & 0.049 & 0.492 & 0.292 & 0.002 \\
\hline Acidosis & -0.192 & 0.006 & 0.4 & $<0.001$ \\
\hline Alkalosis & 0.065 & 0.357 & 0.292 & 0.002 \\
\hline Acute kidney injury & -0.007 & 0.924 & 0.23 & 0.017 \\
\hline Hyperkalemia & -0.032 & 0.649 & 0.153 & 0.113 \\
\hline Hypokalemia & -0.043 & 0.548 & 0.215 & 0.025 \\
\hline Hypernatremia & 0.092 & 0.193 & - & - \\
\hline Hyponatremia & -0.076 & 0.258 & -0.036 & 0.715 \\
\hline Shock & -0.075 & 0.292 & 0.208 & 0.031 \\
\hline Gastrointestinal bleeding & -0.035 & 0.618 & 0.153 & 0.113 \\
\hline Disease severity & -0.02 & 0.841 & - & - \\
\hline Respiratory support & -0.136 & 0.086 & -0.589 & $<0.001$ \\
\hline
\end{tabular}

Bold values mean these varibles were statistically significant 
Table 7 This table shows the association between vital signs and laboratory investigations and length of stay and severity of the disease

\begin{tabular}{|c|c|c|c|c|}
\hline \multirow[t]{3}{*}{ Variables } & \multicolumn{4}{|c|}{ Correlation } \\
\hline & \multicolumn{2}{|c|}{$\begin{array}{l}\text { Length of } \\
\text { in-hospital stay }\end{array}$} & \multicolumn{2}{|c|}{$\begin{array}{l}\text { Severity of the } \\
\text { disease }\end{array}$} \\
\hline & $R$ & $P$-value & $R$ & $P$-value \\
\hline Heart rate & -0.078 & 0.323 & 0.276 & 0.003 \\
\hline Systolic blood pressure & 0.114 & 0.141 & -0.034 & 0.723 \\
\hline Diastolic blood pressure & -0.048 & 0.539 & -0.354 & $<0.001$ \\
\hline Respiratory rate & 0.056 & 0.5789 & 0.544 & $<0.001$ \\
\hline Temperature & -0.033 & 0.765 & 0.135 & 0.332 \\
\hline $\mathrm{SpO}^{2}$ & -0.063 & 0.429 & -0.73 & $<0.001$ \\
\hline WBCs counts & -0.08 & 0.344 & 0.551 & $<0.001$ \\
\hline Monocyte count & -0.038 & 0.72 & 0.192 & 0.128 \\
\hline Lymphocyte count & 0.001 & 0.988 & -0.439 & $<0.001$ \\
\hline Neutrophil count & -0.029 & 0.755 & 0.651 & $<0.001$ \\
\hline Red blood cells counts & -0.091 & 0.346 & -0.511 & $<0.001$ \\
\hline Haemoglobin level & -0.03 & 0.72 & -0.496 & $<0.001$ \\
\hline Hematocrit & -0.118 & 0.215 & -0.542 & $<0.001$ \\
\hline Platelet counts & 0.103 & 0.23 & -0.177 & 0.1 \\
\hline CRP level & -0.01 & 0.922 & 0.718 & $<0.001$ \\
\hline ESR level & 0.127 & 0.34 & 0.419 & 0.004 \\
\hline Ferritin & 0.251 & 0.301 & 0 & 1 \\
\hline Lactate dehydrogenase & 0.263 & 0.292 & 0.316 & 0.604 \\
\hline Random blood sugar & -0.073 & 0.472 & 0.441 & $<0.001$ \\
\hline Sodium & 0.252 & 0.005 & 0.321 & 0.005 \\
\hline Potassium & -0.041 & 0.651 & 0.042 & 0.72 \\
\hline Blood urea & 0.026 & 0.765 & 0.435 & $<0.001$ \\
\hline Creatinine & 0.132 & 0.131 & 0.293 & 0.006 \\
\hline Alanine aminotransferase & -0.04 & 0.74 & 0.427 & 0.002 \\
\hline aspartate aminotransferase & -0.043 & 0.73 & 0.65 & $<0.001$ \\
\hline Alkaline aminotransferase & 0.232 & 0.063 & 0.588 & $<0.001$ \\
\hline Total bilirubin & 0.105 & 0.471 & 0.349 & 0.047 \\
\hline D-dimer & 0.405 & 0.056 & -0.826 & $<0.001$ \\
\hline
\end{tabular}

Bold values mean these varibles were statistically significant

frequency. General manifestations including fever and fatigue were reported in $(83.2 \%)$ and $(33.5 \%)$ of the cases. Our study identified that gastrointestinal symptoms were presented in $(17.3 \%)$ of the patients, and diarrhea was the most commonly mentioned symptom, found in $42.9 \%$ of the participants, followed by nausea and vomiting (35.7\%) and abdominal pain (30.9\%). Similarly, Ramachal et al. reported diarrhea as the most common GI manifestation (14.7\%), followed by nausea and vomiting (10.7\%) and abdominal pain (2\%) [24]. Also, Assiri et al. and Dawei et al. showed that diarrhea was the predominant GI symptom (22\% and $10.1 \%)$, respectively $[25,26]$.
Additionally, neurological manifestations have been reviewed in this study, ranging from mild non-specific neurological symptoms to more severe ones. Headache was the most common neurological manifestation (32.1\%). These findings were consistent with the previous study conducted in China (7). No documented CT brain reports were found in the examined files.

In terms of vital signs, the median for heart rate was 90 beats/min $(n=189)$, respiratory rate 24 breaths/ $\min (n=116)$, temperature $37(n=39)$ and $\mathrm{SpO}^{2} 95 \%$ $(n=182)$. The median systolic and diastolic blood pressures were 131 and 80 , respectively. Of 243 patients, about $65.8 \%$ had high systolic blood pressure compared to $30.1 \%$ with normal blood pressure.

The majority of patients (75\%) did not show any complications during the course of the disease. However, among the 243 patients, the most common complication was sepsis $(9.9 \%)$, followed by acidosis $(7.8 \%)$, respiratory failure (6.9\%), AKI (6.2\%), and ARDS (4.1\%). With regard to the current study, sepsis and acidosis were the most commonly mentioned complications. In contrast, Chen et al. showed that ARDS and respiratory failure were the most common complications [19]. Therefore, early identification and proper treatment of critical cases are of high importance. As Sudan is a low-income country with a weak health system and poverty, patients cannot afford investigations such as blood cultures to identify organisms causing sepsis; hence, health care workers depend only on the patient's clinical situation for assessment and choosing the proper antibiotics.

Among all reported symptoms, shortness of breath (SOB) and cough were the most prevalent symptoms among moderate and severe groups. Shortness of breath was reported in 137/198 (69.2\%) of the studied patients, with a significant difference among patients, being most prevalent among severe ones $(p \leq 0.00001)$, indicating that $\mathrm{SOB}$ has a predictive value on disease severity. These findings are similar to a study conducted in China [27]. Headache and sore throat were documented in $(23.1 \%$ and $16.2 \%)$ and are associated with mild and moderate COVID-19. $(p \leq 0.00001)(p \leq 0.011)$, respectively.

Regarding the outcome, most admitted patients stayed for 7 days or less, $9.8 \%$ of patients required ICU admission. Also, 131 (67.9\%) patients were discharged home compared to $34(17.6 \%)$ who died. The mortality is considered of a high rate, and this can be explained by several factors, the most important of which is the worsening economic situation of the country, as this hinders the expansion of diagnosis, tracing the cases and contacts, doing investigations, and proper management of the cases. Another factor is drug insecurity, either unavailability of essential drugs or unaffordability of the price by the patients [28]. It is worth mentioning that low medical 
resources, shortage of personal protective equipment for HCWs, and deteriorating healthcare system directly influence patients' care and survival. Furthermore, lack of commitment with precautions in the community like wearing masks and social distance, and late presentation of the patients to hospitals have a role in this alarming rate.

This study is considered the first multicenter observational study to describe the clinical manifestations, laboratory investigations, and outcomes for patients with COVID-19 in Sudan and one of the fewest studies in Africa. Additionally, it gives a detailed description and new information for all general, neurological, gastrointestinal, pulmonary, and vascular symptoms and investigations for patients with COVID-19.

However, our study had several limitations. First, it is a retrospective study based on documented files; therefore, although we collected many files, we excluded more than 200 files due to incomplete necessary information. We recommend that documentation practices among health care providers must be evaluated on an ongoing basis using audits. Also, an electronic entry system should be established. Second, we excluded all patients diagnosed and treated as COVID-19 based on their radiological findings only without RT-PCR in order to obtain more accurate and precise results, but we found that laboratory testing was not available for many patients, most probably due to financial reasons; this contributed to further losses in the numbers of patients. Third, we undoubtedly lost patients who had mild symptoms, diagnosed and treated in an outpatient setting.

\section{Conclusion}

Among the general manifestations for patients diagnosed with COVID-19, fever was the most mentioned sign, followed by fatigue. Shortness of breath and cough were the most commonly recorded pulmonary symptoms manifested. Most of the patients did not show any in-hospital complications. Several factors were found associated with in-hospital death. The mortality rate was high, and it increased in severe and critically ill patients diagnosed with COVID-19.

\footnotetext{
Abbreviations

ACE: Angiotensin-converting enzyme 2; AKI: Acute kidney injury; ARDS: Acute respiratory distress syndrome; CFR: Case fatality risk/ratio; COVID19: Coronavirus disease 2019; CRP: C-reactive protein; CVD: Cardiovascular diseases; ESR: Erythrocyte sedimentation rate; Gl: Gastrointestinal; HDU: High dependency unit; ICU: Intensive care unit; IPC: Integrated Food Security Phase Classification; LDH: Lactate dehydrogenase; MERS-Cov: Middle east respiratory syndrome coronavirus; PT: Prothrombin time; PTT: Partial thromboplastin time; RBC: Red blood cell; RT-PCR: Reverse transcription-polymerase chain reaction; SARS-Cov-2: Severe acute respiratory syndrome coronavirus 2; SD: Standard deviation; SOB: Shortness of breath; SpO2: Saturation of peripheral oxygen; SPSS: Statistical Package for the Social Sciences.
}

\section{Acknowledgements} Not applicable.

\section{Authors' contributions}

EAH, FAA, SAMAE, WKK, YTA, MSM, YAM: idea conception, study design. FAA, SAMAE, MKN, WKK, HAM, LAA, SAH, AMT, EHAE, NHK, SAM, SOE, OOA, DAMA: data collection and investigation. EAH, TAA: data creation, analysis, and interpretation. EAH, FAA, SAMAE, TAA, MKN, SB, YTA: manuscript writing. HA, EMM: supervision. All authors read and approved the final manuscript.

\section{Funding}

Not applicable.

\section{Availability of data and materials}

The datasets used and analyzed during this study are available from the corresponding author on reasonable request.

\section{Declarations}

Ethics approval and consent to participate

Ethical approval had been taken from the national health research ethics committee, Sudan's federal health ministry (27.08.2020/ certificate number: 4-7-20). Permission was obtained from hospital administrators and statistics offices of each hospital to outreach the patients' records. Study participant's consent was not required as the study did not use identifiable data. The data collected for this research will not be used for any other purposes.

\section{Consent for publication}

Not applicable.

\section{Competing interests}

The authors declare that they have no competing interests.

\section{Author details}

${ }^{1}$ Faculty of Medicine, University of Khartoum, ElQasr Avenue, Khartoum, Khartoum state 11111, Sudan. ${ }^{2}$ School of Medicine, Ahfad University for Women, Khartoum, Sudan. ${ }^{3}$ Faculty of Medicine, National Ribat University, Khartoum, Sudan. ${ }^{4}$ Faculty of Medical Laboratory, University of Alzaiem Alazhari, Khartoum, Sudan. ${ }^{5}$ Faculty of Pharmacy, Omdurman Islamic University, Khartoum, Sudan. ${ }^{6}$ Sudan Medical Specialization Board, Khartoum, Sudan. ${ }^{7}$ Federal Ministry of Health, Khartoum, Sudan. ${ }^{8}$ Department of Community Medicine, Faculty of Medicine, University of Khartoum, Khartoum, Sudan.

Received: 11 July 2021 Accepted: 2 November 2021

Published online: 14 November 2021

\section{References}

1. Coronavirus disease (COVID-19). (cited 2021 Jan 26). https://www.who. int/emergencies/diseases/novel-coronavirus-2019.

2. Wang D, Hu B, Hu C, Zhu F, Liu X, Zhang J, et al. Clinical characteristics of 138 hospitalized patients with 2019 novel coronavirus-infected pneumonia in Wuhan, China. JAMA. 2020;323:1061-9. https://doi.org/10.1001/ jama.2020.1585.

3. Coronavirus Disease 2019 (COVID-19)|CDC. (cited 2020 May 14). https:// www.cdc.gov/coronavirus/2019-ncov/index.html.

4. Wu C, Chen X, Cai Y, Xia J, Zhou X, Xu S, et al. Risk factors associated with Acute Respiratory Distress Syndrome and death in patients with coronavirus disease 2019 pneumonia in Wuhan, China. JAMA Intern Med. 2019;2020:1-10. https://doi.org/10.1001/jamainternmed.2020.0994.

5. Young BE, Ong SWX, Kalimuddin S, Low JG, Tan SY, Loh J, et al. Epidemiologic features and clinical course of patients infected with SARS-CoV-2 in Singapore. JAMA. 2020;323:1488-94. https://doi.org/10.1001/jama.2020. 3204.

6. Fan BE, Chong VCL, Chan SSW, Lim GH, Lim KGE, Tan GB, et al. Hematologic parameters in patients with COVID-19 infection. Am J Hematol. 2020. https://doi.org/10.1002/ajh.25774

7. Yang X, Yu Y, Xu J, Shu H, Xia J, Liu H, et al. Clinical course and outcomes of critically ill patients with SARS-CoV-2 pneumonia in Wuhan, China: a 
single-centered, retrospective, observational study. Lancet Respir Med. 2020;8:475-81. https://doi.org/10.1016/S2213-2600(20)30079-5.

8. Matsushita K, Ding N, Kou M, Hu X, Chen M, Gao Y, et al. The relationship of COVID-19 severity with cardiovascular disease and its traditional risk factors: a systematic review and meta-analysis. Glob Heart. 2020;15:64. https://doi.org/10.5334/gh.814.

9. Sudan: WHO Coronavirus Disease (COVID-19) Dashboard IWHO Coronavirus Disease (COVID-19) Dashboard (cited 2021 Jan 26). https://covid19. who.int/region/emro/country/sd.

10. COVID- 19 Case Management Protocols. (cited 2020 Jun 1). http://fmoh gov.sd/index.php/files/download/396.

11. Huang C, Wang Y, Li X, Ren L, Zhao J, Hu Y, et al. Clinical features of patients infected with 2019 novel coronavirus in Wuhan, China. Lancet. 2020;395:497-506. https://doi.org/10.1016/S0140-6736(20)30183-5.

12. Richardson S, Hirsch JS, Narasimhan M, Crawford JM, McGinn T, Davidson $\mathrm{KW}$, et al. Presenting characteristics, comorbidities, and outcomes among 5700 patients hospitalized with COVID-19 in the New York City Area. JAMA. 2020;323:2052-9. https://doi.org/10.1001/jama.2020.6775.

13. Lu R, Zhao X, Li J, Niu P, Yang B, Wu H, et al. Genomic characterisation and epidemiology of 2019 novel coronavirus: implications for virus origins and receptor binding. Lancet. 2020;395:565-74. https://doi.org/10.1016/ S0140-6736(20)30251-8.

14. Li W, Sui J, Huang IC, Kuhn JH, Radoshitzky SR, Marasco WA, et al. The $S$ proteins of human coronavirus NL63 and severe acute respiratory syndrome coronavirus bind overlapping regions of ACE2. Virology. 2007:367:367-74. https://doi.org/10.1016/j.virol.2007.04.03.

15. de la Vega R, Ruíz-Barquín R, Boros S, Szabo A. Could attitudes toward COVID-19 in Spain render men more vulnerable than women? Glob Public Health. 2020. https://doi.org/10.1080/17441692.2020.1791212.

16. Mueller AL, McNamara MS, Sinclair DA. Why does COVID-19 disproportionately affect older people? Aging (Albany NY). 2020;12:9959-81.

17. Yang J, Zheng Y, Gou X, Pu K, Chen Z, Guo Q, et al. Prevalence of comorbidities and its effects in coronavirus disease 2019 patients: a systematic review and meta-analysis. Int J Infect Dis. 2020;94:91-5. https://doi.org/ 10.1016/j.jij.2020.03.017.

18. Ma RCW, Holt RIG. COVID-19 and diabetes. Diabet Med. 2020;37:723-5. https://doi.org/10.1111/dme.14300

19. Chen N, Zhou M, Dong X, Qu J, Gong F, Han Y, et al. Epidemiological and clinical characteristics of 99 cases of 2019 novel coronavirus pneumonia in Wuhan, China: a descriptive study. Lancet. 2020;395:507-13. https:// doi.org/10.1016/S0140-6736(20)30211-7.
20. Poignon C, Blaize M, Vezinet C, Lampros A, Monsel A, Fekkar A, et al. Incidence of co-infections and superinfections in hospitalized patients with COVID-19: a retrospective cohort study. Clin Microbiol Infect. 2020;11:1-14.

21. Wang D, Hu B, Hu C, Zhu F, Liu X, Zhang J, et al. Clinical characteristics of 138 hospitalized patients with 2019 novel coronavirus-infected pneumonia in Wuhan, China. JAMA. 2020;323:1061. https://doi.org/10.1001/jama. 2020.1585.

22. Liu K, Fang Y-Y, Deng Y, Liu W, Wang M-F, Ma J-P, et al. Clinical characteristics of novel coronavirus cases in tertiary hospitals in Hubei Province. Chin Med J (Engl). 2020;133:1025-31. https://doi.org/10.1097/CM9.00000 00000000744.

23. Guan W, Ni Z, Hu Y, Liang W, Ou C, He J, et al. Clinical characteristics of coronavirus disease 2019 in China. N Engl J Med. 2020;382:1708-20. https://doi.org/10.1056/NEJMoa2002032.

24. Ramachandran P, Onukogu I, Ghanta S, Gajendran M, Perisetti A, Goyal $\mathrm{H}$, et al. Gastrointestinal symptoms and outcomes in hospitalized coronavirus disease 2019 patients. Dig Dis. 2020;38:373-9. https://doi.org/10. $1159 / 000509774$

25. Mao L, Jin H, Wang M, Hu Y, Chen $S$, He Q, et al. Neurologic Manifestations of hospitalized patients with coronavirus disease 2019 in Wuhan, China. JAMA Neurol. 2020;77:683. https://doi.org/10.1001/jamaneurol.2020. 1127.

26. Wu Y, Xu X, Chen Z, Duan J, Hashimoto K, Yang L, et al. Nervous system involvement after infection with COVID-19 and other coronaviruses. Brain Behav Immun. 2020;87:18-22. https://doi.org/10.1016/j.bbi.2020.03.031.

27. Li J, Chen Z, Nie Y, Ma Y, Guo Q, Dai X. Identification of symptoms prognostic of covid-19 severity: multivariate data analysis of a case series in Henan Province. J Med Internet Res. 2020;22:e19636. https://doi.org/10. 2196/19636.

28. Lucero-Prisno DE, Elhadi YAM, Modber MAA, Musa MB, Mohammed SEE, Hassan KF, et al. Drug shortage crisis in Sudan in times of COVID-19. Public Heal Pract. 2020;1:100060. https://doi.org/10.1016/j.puhip.2020. 100060.

\section{Publisher's Note}

Springer Nature remains neutral with regard to jurisdictional claims in published maps and institutional affiliations.
Ready to submit your research? Choose BMC and benefit from:

- fast, convenient online submission

- thorough peer review by experienced researchers in your field

- rapid publication on acceptance

- support for research data, including large and complex data types

- gold Open Access which fosters wider collaboration and increased citations

- maximum visibility for your research: over 100M website views per year

At BMC, research is always in progress.

Learn more biomedcentral.com/submissions 